\title{
Locating cloud-to-ground lightning flashes with simultaneous two-station measurements
}

\author{
D.U.J. Sonnadara ${ }^{*}$,, A.B. Weerasekera ${ }^{\mathrm{a}}$, I.M.K. Fernando ${ }^{\mathrm{a}}$, R. Lelwala ${ }^{\mathrm{a}}$, \\ K.P.S.C. Jayaratne ${ }^{\mathrm{a}}$, T.R. Ariyaratne $^{\mathrm{a}}$, S. Namasivayam ${ }^{\mathrm{b}}$ and K.R.A. Bandara ${ }^{\mathrm{c}}$ \\ ${ }^{a}$ Department of Physics, University of Colombo, Colombo 3, Sri Lanka \\ ${ }^{b}$ Electro-Technology Unit, CISIR, Colombo 7, Sri Lanka \\ ${ }^{c}$ Department of Meteorology, Colombo 7, Sri Lanka
}

\begin{abstract}
The position reconstruction of the point of strike of cloud-to-ground lightning flashes in several thunderstorms recorded by two-wideband magnetic direction finding stations is presented. Lightning activity data show a clear peak around 18.00 hours, which is a characteristic feature of the Northeast monsoon thunderstorms in Sri Lanka. It is shown that the accuracy of the position reconstruction with two stations is heavily dependent on the difference in the angles measured by the stations. A technique that utilizes the measured strength of the flashes was introduced to improve the accuracy of the position reconstruction. With this technique, the point of strike of ground flashes can be localized with an accuracy of better than $\pm 5 \mathrm{~km}$ within a 100 $\mathrm{km}$ radius around Colombo $(6.90 \mathrm{~N}, 79.86 \mathrm{E})$, except in the vicinity of the line joining the two stations.
\end{abstract}

\section{INTRODUCTION}

Wideband magnetic direction finding (DF) networks are used in many countries to locate and monitor the movement and intensity of lightning activity in thunderstorms as well as in lightning research. These systems are designed primarily to respond to

\footnotetext{
${ }^{*}$ Corresponding author
} 
cloud-to-ground lightning and discriminates against cloud discharges and other background noise ${ }^{1}$.

A DF system senses the electromagnetic fields generated by lightning through two orthogonal magnetic-loop antennas and a flat plate electric antenna. The direction of the lightning flash is determined by the ratio of the strengths of the signals induced on the two orthogonal loops at the time when the radiation field reaches its initial peak (at a height of about few tens of meters from ground level) ${ }^{2}$. This is done in order to minimise the errors associated in direction measurement due to horizontal channel sections, branch currents as well as ionospheric reflections. The electric antenna is used in removing the 180 degree ambiguity in determining the direction of lightning due to positive and negative flashes. To eliminate the interference and reduce background noise, the DF electronics are designed to respond only to those wave shapes that are characteristic of return strokes in cloud-to-ground lightning flashes. A DF unit is capable of providing the time of occurrence, angle of the strike with respect to north, flash strength, number of strokes in a flash (multiplicity) and the polarity of lightning ground flashes.

A network of DF stations is needed in order to locate lightning flashes in and around Sri Lanka. However, before setting up of a network with many DF stations, it is important to understand the behaviour, the instrumental uncertainties and the limitations in DF systems in localising lightning ground flashes. A detailed description of the DF stations used in this work and their performance is described elsewhere ${ }^{3}$. In this paper the results of reconstructing the strike location of lightning ground flashes using two DF stations, the minimum number of stations needed for localising a ground flash, for several thunderstorms during the Northeast monsoon period are presented. A MonteCarlo simulation was deployed to understand the possible errors associated in calculating the point of strike of lightning flashes with respect to the placement of the stations. A technique that is based on the flash strength is used to improve the position reconstruction, especially to overcome the systematic errors in the angular measurement. These errors are considered to be related to the site, i.e. the site errors and other geometric effects with respect to the stations.

\section{DATA \& RESULTS}

During the latter part of the Northeast monsoon period (end of February 1999), two DF stations were utilised to record lightning ground flashes. The stations were installed in observation sites maintained by the Department of Meteorology in Colombo (6.90N, 79.86E) and in Ratnapura (6.68N, 80.40E), located at $7.3 \mathrm{~m}$ and $86.3 \mathrm{~m}$ from sea level respectively. Both electric and magnetic field antennas of the DF units were fixed at a height of $1.5 \mathrm{~m}$ from ground level. The cables connecting the antennas and the 
electronic units were shielded and laid underground to reduce the interference effects due to induced magnetic fields. At each DF station, a personal computer was coupled to the system via a built-in serial port to record the lightning data. A data transfer rate of 1200 bps was used to transfer the data from the DF stations to the PCs. Microsoft NT with Remote Access Server (RAS) was installed on the PCs to allow remote access to lightning data via telecommunication lines. The criterion used to select the lightning strikes is the coincidence in the time of occurrence, since the distance between the stations $(65 \mathrm{~km})$ can only contribute to a time jitter of not more than $0.2 \mathrm{~ms}$.

The recorded data were first screened by rejecting the signals with very low amplitudes or signals that overflowed the DF units ${ }^{3}$. The events that recorded simultaneous hits (coincidences) in both stations were then selected by applying a time window of $100 \mathrm{~ms}$. The pairs of hits that satisfied this selection criterion were marked and identified as true events produced by individual lightning strikes. When selecting coincidence pairs, the recorded hits were allowed to make any number of pairs so long as they fell within the time window. The estimated number of hits that may form false pairs because they fell within this time window for the present data set is not more than $8 \%$. Table 1 shows the total number of coincidences together with the agreement in multiplicity (number of strokes in a flash), and polarity recorded by the two stations.

Table 1: Summery of observations for thunderstorms from February 17,1999 to February 26, 1999.

\begin{tabular}{|c|c|c|c|}
\hline Date & $\begin{array}{c}\text { Coincidence } \\
\text { Strikes }\end{array}$ & $\begin{array}{c}\text { Agreement in } \\
\text { multiplicity \% }\end{array}$ & $\begin{array}{c}\text { Agreement in } \\
\text { polarity \% }\end{array}$ \\
\hline Feb 17, 1999 & 134 & 37.3 & 97.7 \\
Feb 18, 1999 & 47 & 48.9 & 95.7 \\
Feb 19, 1999 & 173 & 55.5 & 97.1 \\
Feb 20, 1999 & 277 & 49.8 & 98.2 \\
Feb 21, 1999 & 153 & 61.6 & 93.9 \\
Feb 22, 1999 & - & - & - \\
Feb 23, 1999 & 45 & 48.8 & 100.0 \\
Feb 24, 1999 & 46 & 30.4 & 95.6 \\
Feb 25, 1999 & 151 & 48.3 & 97.3 \\
Feb 26, 1999 & 48 & 47.9 & 100.0 \\
\hline
\end{tabular}

A total of 1074 hits satisfied the selection criteria and could be considered as genuine lightning ground flashes that occurred during this period. Approximately 55\% of the screened data from Colombo station and $70 \%$ of the screened data from Ratnapura contributed to the coincidences. Table 1 shows that, 
for the recorded lightning strikes, the agreement in the multiplicity between the two DF stations is quite poor. However, the agreement in the polarity of the strikes is very high.

In an ideal situation, both DF stations should register the same polarity and identical values for the multiplicity for any given lightning flash. The percentage of the difference in the multiplicity for the two DF stations is shown in Fig 1a. The data show that on the average, in $50 \%$ of the events both DF stations provide the same multiplicity. In about $25 \%$ of the events it differs by \pm 1 , and in $25 \%$ of the cases the difference in multiplicity was more than \pm 1 . These numbers are quite different from the numbers extracted in a previous study done by Fernando et al. ${ }^{3}$ keeping the two DF stations in the same location. They obtained $70 \%, 20 \%$ and $10 \%$ for the same. In that study they have attributed the disagreement between the two DF stations to the difference in the hardware settings in identifying the multiple strokes in a single flash since only instrumental errors can contribute to their measurement. The additional reduction in the coincidence measured in this study can be attributed to the difference in attenuation of the signal strength received by the two DF stations ${ }^{4,5}$. The strengths of different strokes in lightning flashes can vary from flash to flash. In some cases strokes producing induced voltage pulses of very small amplitudes can be above the trigger thresholds of DF units in one station which is situated closer to the point of strike while the amplitudes may not be large enough to pass the trigger thresholds in a distant station. Since the stations are $65 \mathrm{~km}$ away from each other, propagation effects caused by finitely conducting ground can easily affect the multiplicity measurement and can vary from thunderstorm to thunderstorm depending on the distance from each station.
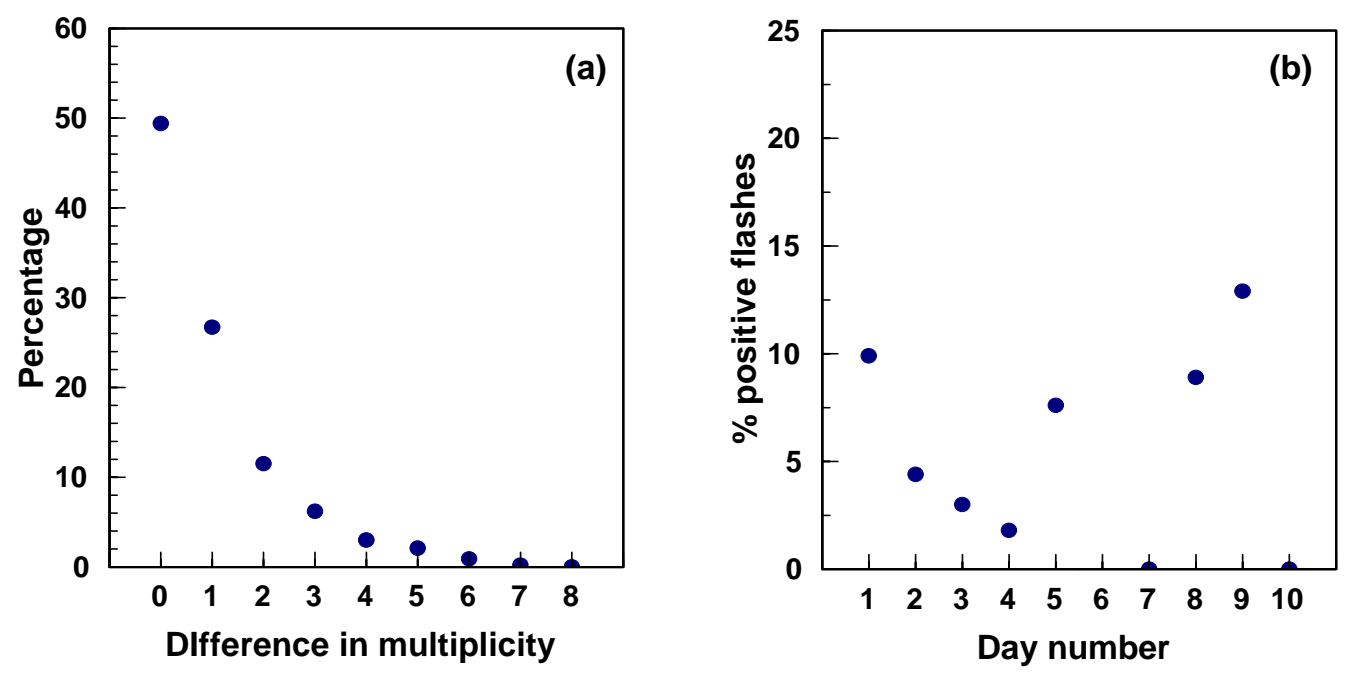

Fig. 1: (a) Difference in multiplicity reported by two-station (b) Percentage of positive flashes. 
The daily variation of the percentage of positive flashes during the above mentioned period are shown in Fig 1b. The percentage varies between $0 \%$ and $13 \%$ for these thunderstorms. It was observed that, of the total number of identified flashes, the DF stations failed to report the correct polarity only in $3 \%$ of the events. Our measurements indicate that multiplicity is a poor parameter to be used as a criterion for selection of coincidence strikes. However, the above observation indicates that the reliability of the data can be enhanced by employing the polarity as an additional criterion for the selection of lightning ground flashes although this selection was not used in the present work.

Figure 2 shows the histogram of lightning activity per hour recorded by the DF stations from February 17,1999 to February 26, 1999. The histogram shows a clear peak around 18.00 hours. This is a characteristic feature of the thunderstorms during the Northeast monsoon season in Sri Lanka, particularly when the low and middle tropospheric wind streams in the Bay of Bengal region blows from east to west ${ }^{6}$. Under the influence of this condition, fair weather prevails over the island in the morning leading to surface heating by solar radiation. Convection together with orography cause the development of thunderclouds over the central hills during the afternoon hours. These thunderclouds are later pushed to the western regions by the easterly winds. Due to this reason, thunderstorms are very common over the western parts during the late evening and early night hours. The development of thunderclouds along the western coastline is likely to be enhanced by the convergence of the easterly wind flow and the sea breeze during the evening. Although high activity is expected during the evening and night hours for the Northeast monsoon rains, this is the first occasion when the lightning activity has been systematically studied and quantitative results are produced to show the link between the two activities in Sri Lanka. From the total detected ground flashes, $94 \%$ of the flashes were observed between $12.00-24.00$ hours.

Fig. 2: Lightning activity observed from February 17, 1999 to February 26, 1999.

\section{MONTE-CARLO SIMULATION STUDIES}

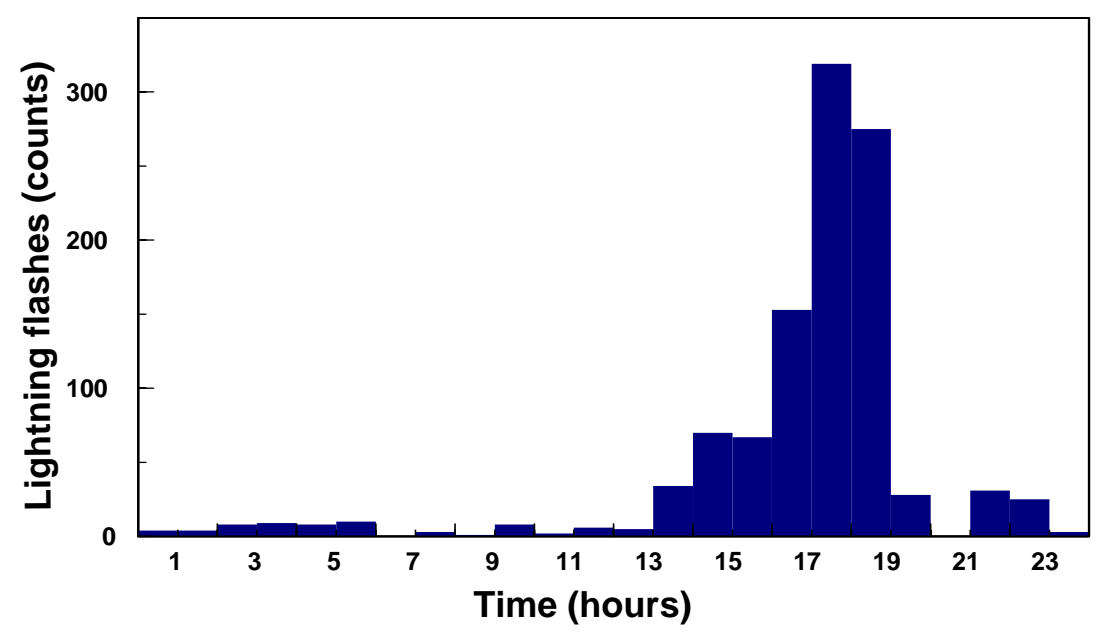


The angular accuracy plays an important role in reconstructing the location of the ground flashes. The two major factors that could influence the angular accuracy are the site errors (errors caused by the external objects in the vicinity of the antennas that can produce induced magnetic fields), and the instrumental errors. The site errors can be minimised by installing the DF stations in locations where there are no nearby metallic objects. Ratnapura and Colombo meteorology stations were chosen especially for this reason. The authors have reported the instrumental errors associated with the present system in an earlier study ${ }^{3}$. With two DF stations, the position of a ground flash is merely the crossing point of the two position vectors subtended by the two stations. Hence, any systematic or instrumental errors associated with the angular measurement directly influence the position accuracy when only two systems are used.

To understand the possible errors in two station measurements, a Monte-Carlo program was utilized ${ }^{7}$. It generates the angle to the point of strike, and the signal strength at each DF station convoluted with measured instrumental uncertainties of $\pm 0.5^{\circ}$ and $\pm 1.5 \%$ respectively. A normal distribution was assumed in generating the uncertainties. The position of the lightning strike was varied randomly. The locations of the DF stations were defined using the corresponding longitudes and latitudes. The exact positions of the strikes were compared with the reconstructed positions using the simulated information obtained from the two stations placed at Colombo and at Ratnapura.
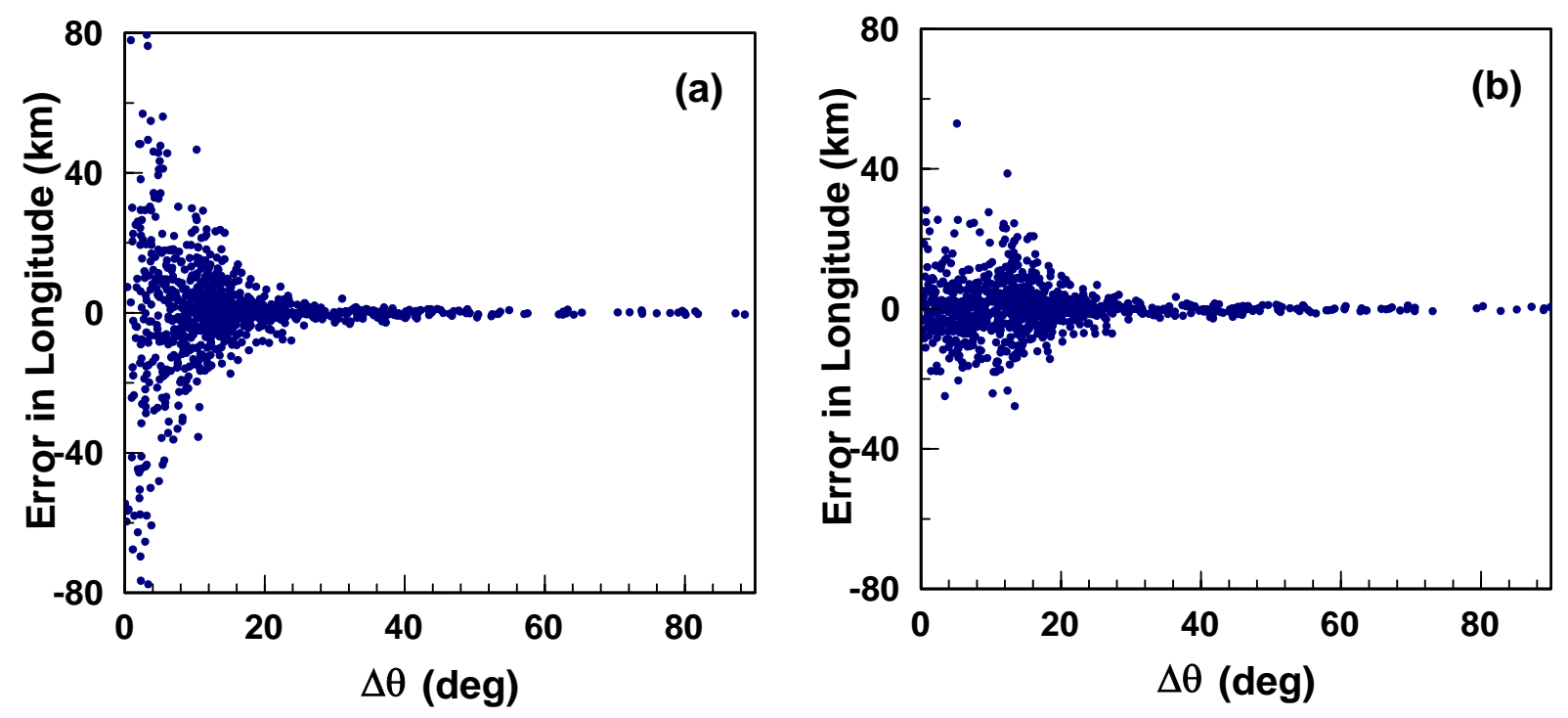

Fig 3: (a) Difference between the reconstructed position and the simulated position vs. the difference in angle $(\Delta \theta)$ observed by the two stations (b) Same parameters but after applying the signal strength correction discussed in the text.

Fig 3a shows the difference between the reconstructed position and the simulated position vs. the difference in the angle observed by the two stations. The angles greater than 90 degrees were re-mapped onto the same scale $\left(0^{\circ}-90^{\circ}\right)$ by subtracting from 180 degrees since they show the same behavior. It can be seen that, when the difference in 
angles is greater than 25 degrees, the lightning strikes can be localized with an accuracy of $\pm 2 \mathrm{~km}$. As expected, angular measurements obtained from two stations show large dispersions when the difference in angles is smaller. The measured width of the distribution for difference in angles $(\Delta \theta)$ below 10 degree is $\pm 30 \mathrm{~km}$.

The possibility of using signal strengths to enhance the accuracy of the position reconstruction has also been explored. If the true source strength is $\mathrm{S}$ and the signal strengths seen by the two stations are $S_{1}$ and $S_{2}$, then by assuming flat uniformly conducting ground, the distances $r_{1}$ and $r_{2}$ to the point of strike from the two DF stations can be related to other parameters by,

$$
\mathrm{S}=\mathrm{S}_{1} \times \mathrm{r}_{1}\left(\theta_{1}, \theta_{2}\right)=\mathrm{S}_{2} \times \mathrm{r}_{2}\left(\theta_{1}, \theta_{2}\right)
$$

where $r_{1}$ and $r_{2}$ are functions of the measured angles $\theta_{1}$ and $\theta_{2}$ respectively at the two DF stations. Hence, by varying the angles within their instrument uncertainties one can minimize the function

$$
f\left(\theta_{1}, \theta_{2}\right)=\mathrm{S}^{\prime}-\mathrm{S}^{\prime \prime}
$$

to obtain new coordinates of the intersection of the position vectors, which in principle should provide a better estimate for the point of strike of the lightning. Here $S^{\prime}$ and $S^{\prime \prime}$ represent the source strengths measured by the DF stations which are given by the expressions

$$
\mathrm{S}^{\prime}=\mathrm{S}_{1} \times \mathrm{r}^{\prime}\left(\theta_{1}, \theta_{2}\right) \quad \text { and } \quad \mathrm{S}^{\prime \prime}=\mathrm{S}_{2} \times \mathrm{r}^{\prime \prime}\left(\theta_{1}, \theta_{2}\right)
$$

and $r^{\prime}$ and $r^{\prime \prime}$ are the new position vectors of $r_{1}$ and $r_{2}$ that result from the above mentioned minimisation process. Fig $3 b$ shows the reconstructed positions obtained after applying the above technique to the same set of points shown in 3a.

Fig $3 \mathrm{~b}$ shows that below 10 degrees, the spread in the distribution is now much smaller. Although this technique will not bring the spatial resolution of the position reconstruction to within 1-2 km for the data below 10 degrees, it avoids position reconstruction from reporting values which are very far from the true position, specially for strikes that fall in the line joining the two stations. The measured width of the distribution for $\Delta \theta$ below 10 degrees turned out to be $\pm 10 \mathrm{~km}$, which is better by a factor of 3 compared to the direct reconstruction.

In order to investigate the position error associated with the point of strike within the region of the Indian Ocean where Sri Lanka is located, the area bounded by $5.0 \mathrm{~N}$, $78.0 \mathrm{E}$ and $11.0 \mathrm{~N}, 83.0 \mathrm{E}$ was divided into $24 \times 20$ cells (each cell corresponding to an area of $28 \times 28 \mathrm{~km}^{2}$ ) and the position error was calculated within each cell for 1000 randomly selected hits. In this study the DF stations were considered to be fixed at locations corresponding to Colombo and Ratnapura. Fig 4 shows the contour plot of position 
errors corresponding to the above region. The slight asymmetry in the contour plot with respect to the stations is caused due to the selection of the grids in calculating the errors. The graph shows that a position accuracy of $\pm 5 \mathrm{~km}$ can be achieved in the region of Colombo where major industries are located except in the vicinity of the line joining the two stations. Most parts of Sri Lanka can be covered under a $\pm 10 \mathrm{~km}$ accuracy except for the extreme north. The errors in the vicinity of the line joining the two stations can be eliminated by installing a third station not in line with the existing stations.

Fig 4: Position error distribution for the region where Sri Lanka is located. The two stations are placed at Colombo and Ratnapura.

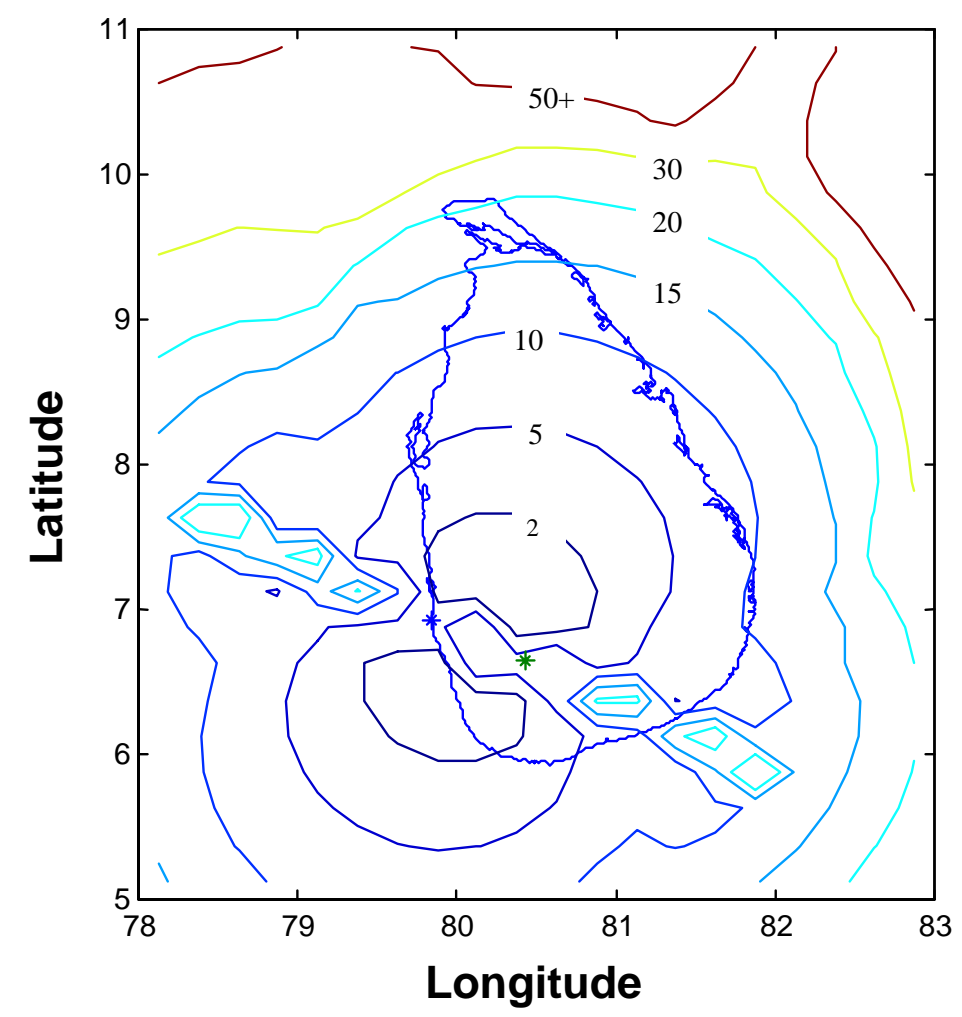

\section{RECONSTRUCTION OF STRIKE LOCATION}

For the flash data the strike position was first reconstructed using the crossing point of the two direction vectors. If the strike position is correct, then the normalized 
signal strengths should show a tight correlation (assuming a uniform propagation path) since it would represent the source strength. In figure $5 \mathrm{a}$, the correlation of the flash strengths measured by the two DF stations are shown. The signal strengths are normalized to $100 \mathrm{~km}$ by multiplying the flash strength by the distance to the strike and dividing by 100 . Only hits that corresponding to a angular difference of greater than \pm 25 degrees at the DF stations are shown in the figure. Although the data show some correlation, it is not as tight as expected and show a large scatter which is well beyond the instrumental resolution of DF stations.

In Figure 5b, the same variables are shown. However, in this case, the data are taken from a measurement where the same DF stations were used to record lightning flashes keeping them at the same location ${ }^{3}$. Data show a very tight correlation since only instrumental errors contribute to the width of the band in this case. Figure 5a clearly demonstrate the limitation in considering the angles recorded at DF stations as the only parameter for calculating the locations of strikes. Observing a large dispersion in 5a can be attributed to two reasons. They are the site errors and the non-uniform propagation path conditions. Site errors can be minimized by means of a transmitter which could produce electromagnetic signals having similar characteristics as those of lightning strikes ${ }^{8}$.
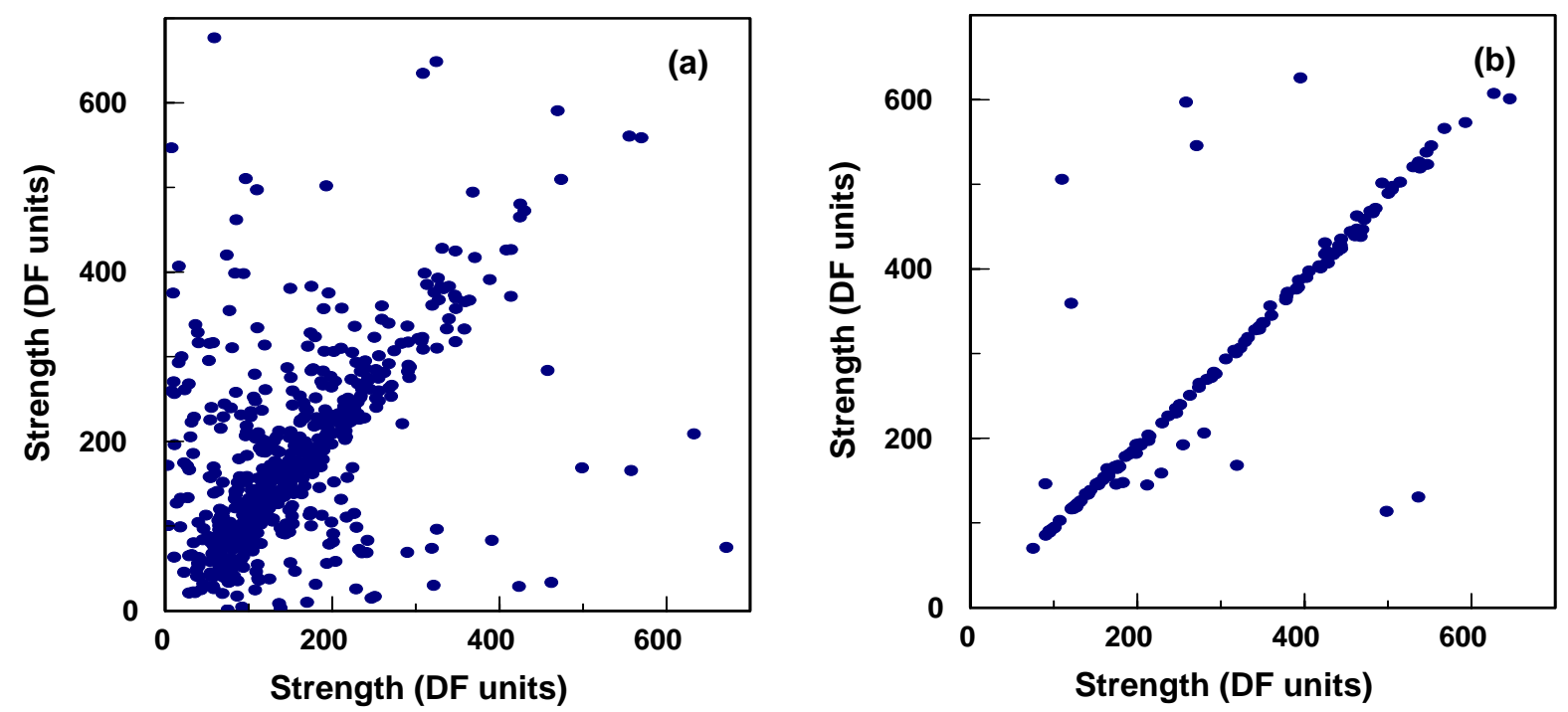

Fig 5: (a) Correlation of normalised (100 km) flash strength measured by the two DF stations for hits that produce an angular difference greater than \pm 25 degrees (b) Signal strengths measured keeping the DF stations in the same location.

To correct the erroneous angular measurement when site errors and other systematic errors are present, the technique tested with the Monte-Carlo simulation can also be utilized. Since all these errors mainly contribute to systematic change in the angular measurement, one can use this technique to calculate the strike position even 
when these errors are present. However, since site errors can contribute to large variations in angular measurement ${ }^{\mathbf{8}}$ the minimization algorithm was allowed to vary angles to within \pm 10 degrees around the measured values, which is well beyond their instrumental uncertainties. Out of 1073 coincidences, 4\% of the hits failed to converge under this criterion and could not be used in the position reconstruction.

Reconstructed ground flash distribution for the data is shown in Figure 6. The locations of the Colombo and Ratnapura DF stations are also shown (open circles). It can be seen that, for the recorded data, most of the activities were concentrated between the latitudes $6.4 \mathrm{~N}$ and $7.2 \mathrm{~N}$ and longitudes $79.8 \mathrm{E}$ and 80.4E (i.e. around Colombo) where most of the industries are located. This area located within a zone corresponds to a position accuracy of $\pm 5 \mathrm{~km}$ in the worst case (see figure 4).

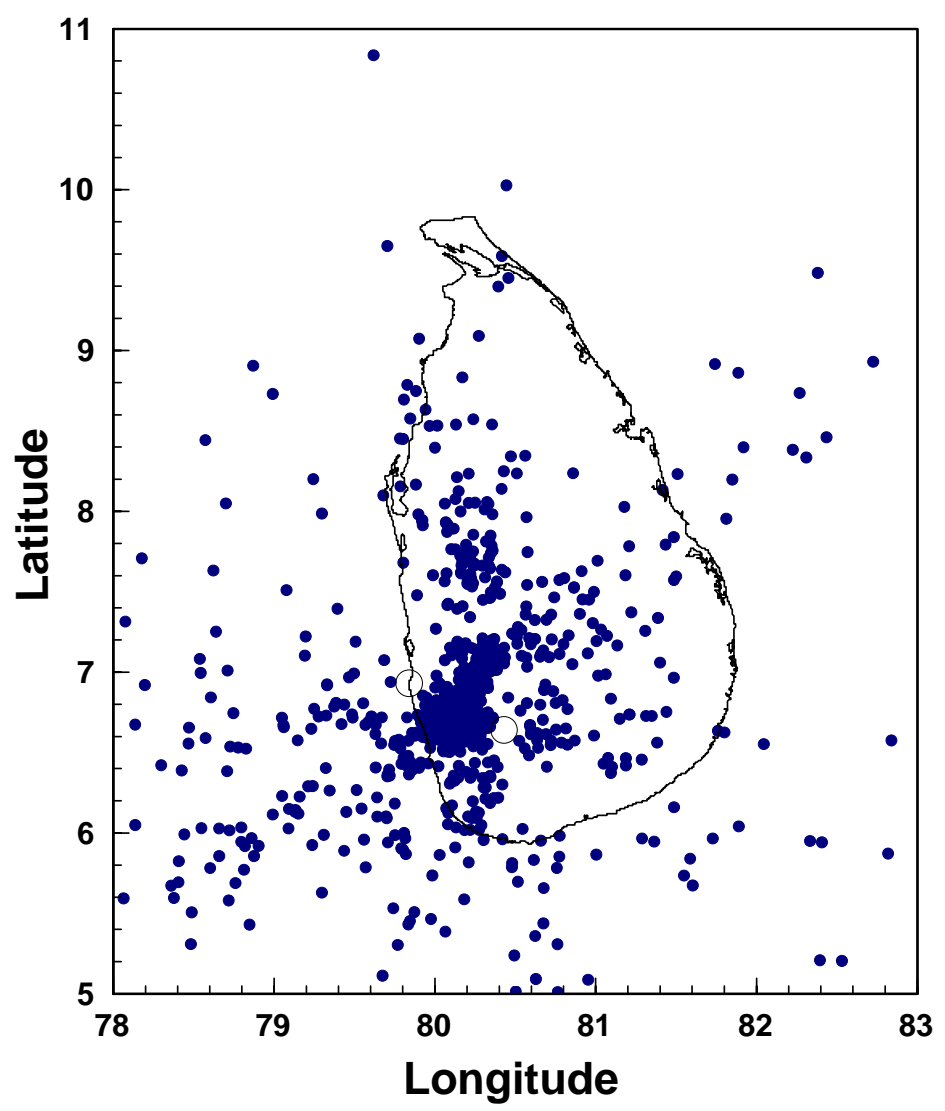

Fig 6: Reconstructed ground flash data for the Northeast monsoon

An attempt has been made to verify the locations of the strikes given by the DF stations against the Meteorology Department satellite images generated by the NOAA. In general, the images indicated a heavy cloud coverage in the regions in which the DF stations indicated considerable lightning activity. However, the cloud images generated 
by the satellite are not suitable for verifying individual lightning strikes since the extent of the cloud images detected by the satellite are vast.

\section{SUMMARY \& CONCLUSIONS}

The position reconstruction of lightning ground flash data for several Northeast monsoon thunderstorms are presented. Less than $8 \%$ of accidentals are observed after using an algorithm based on a $100 \mathrm{~ms}$ time window in selecting the coincidence strikes between the two DF stations that are kept $65 \mathrm{~km}$ apart. Only $3 \%$ of the selected flashes show disagreement in polarity and it can be used as an additional criterion to screen ground flashes. Multiplicity of the lighting flashes differs from one DF station to another, and hence cannot be used as a reliable criterion to identify single events. About $50 \%$ of the recorded flashes produced the same multiplicity while $25 \%$ showed a difference of \pm 1 .

It is also revealed that the position reconstruction through the angular information alone could lead to large errors especially when site errors and other topographical errors with respect to the locations of the stations are concerned. A technique that utilises the measured strengths of the lightning flashes was introduced successfully in the position reconstruction of strikes. This technique can be used to reconstruct the position of lightning strikes within an accuracy of $10 \mathrm{~km}$ inside Sri Lanka except for the extreme north of the island.

\section{ACKNOWLEDGEMENTS}

The authors wish to thank the International Program in Physical Science (IPPS) of the University of Uppsala, Sweden for donating the LLP stations and other equipment. Financial assistance by IPPS, Uppsala University, Sweden (research grant number SRI:01/1), The National Science Foundation, Sri Lanka (grant number RG/99/P/02), and University of Colombo, Sri Lanka (grant number 99/S/31) are also acknowledged.

\section{REFERENCES}

1. Direction-Finding System Operating Manual, LLP Inc. (1979)

2. Tests of the principle of operation of a wideband magnetic direction finder for lightning return strokes, B.D. Herrman, M.A. Uman, R.D. Brantley and E.P. Krider, J. Appl. Meteor., 15, pp $402-405$ (1976) 
3. Study of the performance of direction finding stations prior to the implementation of a Lightning Location System in Sri Lanka, I.M.K. Fernando, D.U.J. Sonnadara, K.P.S.C. Jayaratne, T.R. Ariyaratne, S. Namasivayam \& K.R.A Bandara, Ceylon Journal of Science $\underline{5}$, pp 65-73 (1998).

4. Effect of surface conductivity on the peak magnetic field radiated by first return strokes in cloud-toground lightning, L.J. Tyahla and P.E. Lopez, J. Geophys. Res. 99, pp 10517-10525 (1994)

5. Effects of propergation on the rise times and the initial peaks of radiation fields from return strokes, V. Cooray and S. Lundquist, Radio Sci. 18, pp 409-415 (1983)

6. Weather in Sri Lanka, K.R. Abayasinghe, pp 41-44, ISBN 955-95177-16 (1997)

7. The role of site selection on localizing ground flashes in an automated lighting detection network, D.U.J. Sonnadara, I.M.K. Fernando, S. Namasivayam, T.R. Ariyaratne, K.P.S.C. Jayaratne and K.R.A. Bandara, IPSL, Proc. 15th Technical Session, Sri Lanka (1999).

8. An experimental study of the angle correction of the direction finders of the LLP system in Sweden, T. Schutte, Uppsala University, Sweden, UURIE: 158-84 (1984) 